\title{
ADJOINT LINEAR DIFFERENTIAL OPERATORS
}

\author{
BY \\ WILLIAM T. REID
}

1. Introduction. The present paper is concerned with linear differential expressions of the form

$$
L(y) \equiv \sum_{\mu=0}^{n} p_{\mu}(x) y^{(\mu)}(x),
$$$$
n \geqq 1,
$$

with coefficients $p_{\mu}(x)$ belonging to $\&$, the class of complex-valued (Lebesgue) integrable functions of the real variable $x$ on the compact interval $a b: a \leqq x \leqq b$. The symbol $\mathfrak{C}_{k},(k=0,1, \cdots)$, will be used for the class of functions $f(x)$ that are continuous and have continuous derivatives of the first $k$ orders on $a b$, and the subclass of functions $f(x)$ of $\mathfrak{C}_{k-1}$ with $f^{(k-1)}(x)$ absolutely continuous on $a b$ will be designated by $\mathfrak{A}_{k},(k=1,2, \cdots)$; in particular, $\mathfrak{A}_{1}$ is the class of functions absolutely continuous on $a b$. The symbol $\mathfrak{A}_{k ; 2}$ will signify the set of functions $f(x) \in \mathfrak{A}_{k}$ for which $f^{(k)}(x)$ belongs to $\mathfrak{R}_{2}$, the class of square-integrable functions on $a b$. For $k \geqq 1$ the subclasses of functions $f(x)$ of $\mathfrak{S}_{k}, \mathfrak{A}_{k}$ and $\mathfrak{A}_{k ; 2}$ such that $f^{(\alpha)}(a)=0=f^{(\alpha)}(b),(\alpha=0,1, \cdots, k-1)$, will be denoted by $\mathfrak{C}_{k}^{\circ}, \mathfrak{H}_{k}^{\circ}$, and $\mathfrak{U}_{k ; 2}^{\circ}$, respectively. In order to avoid supplementary comments at various places in the paper, it will be understood that the symbols $\mathfrak{H}_{o}$ and $\mathfrak{H}_{0}^{\circ}$ designate the class $\mathfrak{R}$, the symbols $\mathfrak{H}_{0 ; 2}$ and $\mathfrak{H}_{o ; 2}^{\circ}$ denote the class $\mathfrak{R}_{2}$, and that $\mathfrak{C}_{o}^{\circ}$ designates the class $\mathfrak{G}_{o}$ of functions continuous on $a b$. As is customary, functions $f_{1}(x), f_{2}(x)$ that are equal a.e. (almost everywhere) are considered as equal, and we write $f_{1}=f_{2}$; correspondingly, for subsets $\mathfrak{D}_{\alpha} \subset \mathfrak{R},(\alpha=1,2)$, we write $\mathfrak{D}_{1}=\mathfrak{D}_{2}$ if for each $w(x) \in \mathfrak{D}_{\alpha},(\alpha=1,2)$, there is a $u(x) \in \mathfrak{D}_{\beta}, \quad(\beta \neq \alpha)$, such that $u=w$. If $L_{\alpha}(y)=\sum_{\mu=0}^{n} p_{\mu, \alpha}(x) y^{(\mu)},(\alpha=1,2)$, then $L_{1}(y)=L_{2}(y)$ signifies $p_{\mu, 1}=p_{\mu, 2},(\mu=0,1, \cdots, n)$. Finally, if $u=u(x)$, $v=v(x)$ and $u \bar{v}$ belong to $\&$ the symbol $(u, v)$ is used for $\int_{a}^{b} u \bar{v} d x$; in particular, $(u, v)$ exists if $u, v \in \Omega_{2}$.

As $p_{\mu} \in \mathbb{R},(\mu=0,1, \cdots, n)$, if $y \in \mathfrak{S}_{n}$ then $L(y) \in \mathbb{R}$. The operator $T_{o}$ is defined to have domain $\mathfrak{S}_{n}^{\circ}$ and value $T_{o} y=L(y)$ for $y \in \mathfrak{C}_{n}^{\circ}$. If $D^{*}$ denotes the totality of functions $z(x) \in \mathbb{R}$ with $\bar{z}(x) p_{\mu}(x) \in \Omega,(\mu=0,1, \cdots, n)$, and for which there exists a corresponding $f_{z}=f_{z}(x) \in \mathbb{R}$ such that $(L(y), z)=\left(y, f_{z}\right)$ for all $y \in \mathfrak{S}_{n}^{\circ}$, then the operator $T_{0}^{*}$ with domain $\mathfrak{D}^{*}$ and value $T_{o}^{*} z=f_{z}$ is termed the adjoint of $T_{o}$. In particular, if $p_{\mu} \in \mathfrak{C}_{\mu},(\mu=0, \cdots, n)$, and $p_{n}(x) \neq 0$ on $a b$, then classical results provide the conclusion that $\mathfrak{D}^{*}=\mathfrak{A}_{n}$, and for $z \in \mathfrak{A}_{n}$ the value of $T_{o}^{*} z$ is given by the Lagrange adjoint $\sum_{\mu=0}^{n}(-1)^{\mu}\left(p_{n} z\right)^{(\mu)}$. A very

Presented to the Society, April 14, 1956 under the title $A$ note on adjoint linear differential operators; received by the editors June 14, 1956. 
important instance is the Hilbert space case that occurs when $p_{\mu} \in \Omega_{2}$, $(\mu=0,1, \cdots, n)$, and analogous to the above defined $T_{o}$ one considers the operator with values $L(y)$ on the domain of functions $y \in \mathfrak{H}_{n ; 2}^{\circ}$ such that $L(y) \in \Omega_{2}$. For the considerations of the present paper, however, restriction of attention to this case would be undesirable from the point of view of desired generality.

The central purpose of the present paper is to obtain precise information on the nature of $T_{o}$ and its adjoint $T_{o}^{*}$. The main tool used is a moderate extension of the fundamental lemma of the calculus of variations and certain related results, which are derived in $\$ 2$. Some of the most interesting results on the character of $T_{o}$ and $T_{o}^{*}$ obtained in $\$ 3$ deal with differentiability conditions that must hold for coefficients of (1.1) in case the domain $\mathfrak{D}^{*}$ of $T_{o}^{*}$ is sufficiently large. That such type of condition on $\mathfrak{D}^{*}$ can not imply that $p_{\mu}(x) \in \mathfrak{S}_{\mu},(\mu=0,1, \cdots, n)$, or even that $p_{n}(x) \in \mathfrak{S}_{n}$, is exemplified by the case of (1.1) with $L(y)=\Lambda_{n}(y ; p)$, where we define

$$
\begin{aligned}
\Lambda_{o}(y ; p) & \equiv p(x) y, \quad \Lambda_{2 r}(y ; p) \equiv\left(p(x) y^{(r)}\right)^{(r)}, \\
\Lambda_{2 r-1}(y ; p) & =\frac{1}{2}\left[\left(p(x) y^{(r-1)}\right)^{(r)}+\left(p(x) y^{(r)}\right)^{(r-1)}\right],(r=1,2, \cdots)
\end{aligned}
$$

with the understanding that $p(x) \in \mathfrak{A}_{r}$ in the definition of $\Lambda_{2 r}$ and $\Lambda_{2 r-1}$. Indeed, if for (1.1) we have $L(y)=\Lambda_{n}(y ; p),(n \geqq 1)$, then the fact that $\mathfrak{A}_{n} \subset \mathfrak{D}^{*}$, and $T^{*} z=\Lambda_{n}\left(z ;(-1)^{n} \not\right)$ for $z \in \mathfrak{A}_{n}$, is an immediate consequence of the classical relation

$$
u \Lambda_{n}(v ; p)-(-1)^{n} v \Lambda_{n}(u ; p)=\left[K_{n}(u, v ; p)\right]^{\prime} \quad(n \geqq 1),
$$

for arbitrary $u, v$ of $\mathfrak{A}_{n}$, where $K_{n}(u, v ; p)$ is the bilinear concomitant $\sum_{i, j=1}^{n} u^{(i-1)} K_{i j ; n}(x ; p) v^{(j-1)}$. The most important result of $\S 3$ is that of Theorem 3.2, to the effect that if $g_{r} \equiv x^{\lambda} / \lambda ! \in D^{*},\left(\lambda=0,1, \cdots, k_{n}-1\right)$, where $k_{i}$, $(i=0,1, \cdots)$, is equal to $i / 2$ or $(i+1) / 2$ according as $i$ is even or odd, then there exist functions $\pi_{j} \in \mathfrak{A}_{k_{i}},(j=0,1, \cdots, n)$, such that $L(y)=\sum_{j=0}^{n} \Lambda_{j}(y$; $\left.\pi_{j}\right)$, while $\mathfrak{A}_{n} \subset \mathfrak{D}^{*}$ and $T_{o}^{*} z=L^{\star}(z)=\sum_{j=0}^{n} \Lambda_{j}\left(z ;(-1)^{j} \bar{\pi}_{j}\right)$ for $z \in \mathfrak{A}_{n}$; moreover, $\pi_{j} \in \mathfrak{A}_{k_{j, 2},}, \quad(j=0,1, \cdots, n)$, if and only if $T_{o}^{*} g_{\lambda} \in \mathfrak{R}_{2}, \quad(\lambda=0,1, \cdots$, $\left.k_{n}-1\right)$, and $p_{j}(x) \in \mathfrak{R}_{2},\left(j=0,1, \cdots, n-k_{n}\right)$.

$\S 4$ is concerned with the case in which there is an $\epsilon_{o}>0$ such that $\left|p_{n}(x)\right|$ $\geqq \epsilon_{o}$ a.e. on $a b$. In particular, for this case it is proved in Theorem 4.2 that $M(z)=\sum_{\nu=0}^{n} q_{\nu}(x) z^{(\nu)}$, with $q_{\nu}(x) \in \mathbb{R},(\nu=0,1, \cdots, n)$, is such that $(L(y), z)$ $=(y, M(z))$ for arbitrary $z \in \mathfrak{C}_{n}, y \in \mathfrak{C}_{n}^{\circ}$ if and only if $g_{n}(x)=(-1)^{n} p_{n}(x)$, and for linearly independent solutions $y_{j}(x)$ and $z_{j}(x),(j=0,1, \cdots, n)$, of $L(y)$ $=0$ and $M(z)=0$, respectively, there exists a corresponding constant matrix $\Delta \equiv\left\|\Delta_{i j}\right\|,(i, j=1, \cdots, n)$, such that $\sum_{i, j=1}^{n}\left\{y_{i}^{(\alpha-1)}(x) \Delta_{i j} \bar{z}_{j}(x)\right]=\delta_{n \alpha} / p_{n}(x)$, $(\alpha=1, \cdots, n)$. This result is an analogue of one obtained by Hamburger [5] for the Sturm-Liouville operators he considered. $\$ 5$ is devoted to the Sturm- 
Liouville case; from the results of the preceding sections we have the characterization of Sturm-Liouville operators given in Theorem 5.1, which shows in particular that if (1.1) is a formal Sturm-Liouville operator in the sense of Hamburger, then $L(y)=\sum_{j=1}^{n} \Lambda_{j}\left(y ; \pi_{j}\right)$, where $\pi_{j} \in \mathfrak{A}_{k_{j ; 2}}$ and $\pi_{j}=(-1)^{\mathfrak{\jmath}} \bar{\pi}_{j}$, $(j=0,1, \cdots, n)$. Theorem 5.3 also provides a proof of the result of Theorem 1 of Hamburger [5] that is considerably simpler in detail than the proof of Hamburger. $\S 6$ presents results on extensions of the operator $T_{o}$; for the Sturm-Liouville case Theorem 6.2 is an improvement on results of Miller [9] and Hamburger [5], as it shows that a hypothesis of incompatibility made by these authors is unnecessary for the result.

2. Auxiliary results. For brevity, if $f(x) \in \mathfrak{R}$ set $S_{o}(x ; f) \equiv f(x)$ and $S_{k+1}(x ; f)$ $=\int_{x_{o}}^{x} S_{k}(t ; f) d t,(a \leqq x \leqq b ; k=0,1, \cdots)$, where $x_{o}$ is some fixed point on $a<x<b$. Moreover, for $k=0,1, \cdots$ the symbol $\mathfrak{B}_{k}$ will denote the set of polynomials $P_{k}(x)$ of degree at most $k$; the symbol $\mathfrak{B}_{-1}$ will designate the class of functions consisting of the single function $P_{-1}(x) \equiv 0$. Basic for proofs of the subsequent sections is the following result, which is a moderate extension of the so-called fundamental lemma of the calculus of variations.

Theorem 2.1. Suppose that $r_{j}(x) \in \mathfrak{R},(j=0,1, \cdots, h \geqq 0)$, and for $k \geqq h$ let $H_{k}$ denote the condition that

$$
I(y) \equiv \int_{a}^{b}\left[\sum_{j=0}^{h} r_{j}(x) y^{(j)}\right] d x=0 \quad \text { for } y \in \mathfrak{c}_{k}^{\circ} .
$$

Then for a given $k \geqq h$ the condition $H_{k}$ holds if and only if there exists a $P_{h-1}(x)$ $\in \Re_{h-1}$ such that $\sum_{j=0}^{h}(-1)^{i} S_{j}\left(x ; r_{h-j}\right)=P_{h-1}(x)$; in particular, $H_{k}$ is equivalent to $H_{l}$ for $k \geqq h, l \geqq h$. If $H_{k}$ holds for some $k \geqq h$ and $r_{i}(x)=0$ for $h-g<i<h$, then there exists a function $f(x) \in \mathfrak{A}_{g}$ such that $r_{h}(x)=f(x)$; also, $f(x) \in \mathfrak{A}_{g ; 2}$ whenever $r_{h-g}(x) \in \mathfrak{R}_{2}$. Moreover, if $H_{k}$ holds for some $k \geqq h$ then for arbitrary $y \in \mathfrak{H}_{h}^{\circ}$ the integral $I(y)$ exists and is equal to zero.

If $y \in \mathfrak{C}_{\boldsymbol{k}}^{\circ},(k \geqq h)$, then we have

$$
I(y)=\int_{a}^{b} r(x) y^{(h)} d x, \text { where } r(x)=\sum_{j=0}^{h}(-1)^{i} S_{j}\left(x ; r_{h-j}\right) ;
$$

indeed (2.2) is identical with (2.1) if $h=0$, and is a ready consequence of integration by parts in case $h>0$. If there is a $P_{h-1}(x) \in \mathfrak{P}_{h-1}$ such that $r(x)$ $=P_{h-1}(x)$ it follows that condition $H_{k}$ holds for $k \geqq h$; in fact this result is a direct application of (1.3) with $n=h, p(x) \equiv 1, u(x) \equiv P_{h-1}(x)$, and $v(x) \equiv y(x)$, since $\Lambda_{h}\left(P_{h-1}(x) ; 1\right) \equiv 0$.

On the other hand, as $H_{k}$ implies $H_{k+1}$, if $H_{k}$ holds for a value $k \geqq h$ then

$$
0=I(y)=(-1)^{k-h+1} \int_{a}^{b} S_{k-h+1}(x ; r) y^{(k+1)} d x, \quad \text { for } y \in \mathfrak{\complement}_{k+1}^{\circ},
$$


where $r(x)$ is as in (2.2). Consequently, (see, for example, Huke [7, Chapter IV]), the customary form of the fundamental lemma implies that there is a polynomial $P_{k}(x) \in \mathfrak{B}_{k}$ such that $S_{k-k+1}(x ; r) \equiv P_{k}(x)$ on $a b$, and differentiation yields $r(x)=P_{h-1}(x) \in \Re_{h-1}$, with $P_{h-1}(x)$ the $(k-h+1)$ st derivative of $P_{k}(x)$. Finally, if $r(x)=P_{h-1}(x)$ and $r_{i}(x)=0$ for $h-g<i<h$, then $r_{h}(x)=f(x)$, where $f(x) \equiv P_{h-1}(x)-\sum_{j=o}^{h}(-1)^{i} S_{j}\left(x ; r_{h-j}\right)$ if $g \leqq h, f(x) \equiv P_{h-1}(x)$ if $g>h$, and in either case $f(x) \in \mathfrak{A}_{g}$; in particular, if $g \leqq h$ and $r_{h-g} \in \mathfrak{R}_{2}$, then the relation $f^{(o)}(x)=P_{h-1}^{(o)}(x)-\sum_{j=0}^{h}(-1)^{i} S_{j-g}\left(x ; r_{h-j}\right)$ implies that $f^{(o)}(x) \in \Omega_{2}$ and $f(x)$ $\in \mathfrak{A}_{g ; 2}$. In all cases the first conclusion of the preceding sentence holds for $g=1$, that is, the validity of $H_{k}$ for some integer $k \geqq h$ implies the existence of a function $f(x) \in \mathfrak{A}_{1}$ such that $r_{h}(x)=f(x)$. For $h=0$ the last statement of the theorem is obvious. If $h \geqq 1$ and $H_{k}$ holds for some $k \geqq h$ then the fact that there is a function $f(x) \in \mathfrak{P}_{1}$ such that $r_{h}(x)=f(x)$ implies that $I(y)$ exists for $y \in \mathfrak{A}_{h}$, and the stated result follows from the validity of (2.2) for $y \in \mathfrak{A}_{h}^{\circ}$.

It is to be remarked that if $\hat{H}_{k}$ denotes the condition that $I(y)=0$ for $y(x)$ an arbitrary polynomial $P(x)$ satisfying $P^{(\alpha)}(a)=0=P^{(\alpha)}(b),(\alpha=0,1, \cdots$, $k-1)$, then by an extension of the fundamental lemma due to Hobson [6], (see also Huke [7, Chapter IV]), it follows that for $k \geqq h$ the condition $\hat{H}_{k}$ is equivalent to $H_{k}$.

COROLlaRY 1. The condition $H_{k}$ of Theorem 2.1 holds for some integer $k \geqq h$ if and only if there exist functions $v_{i}(x) \in \mathfrak{A}_{1},(i=1, \cdots, h)$ such that

$$
v_{h}=r_{h} ; \quad v_{j+1}^{\prime}+v_{j}=r_{j},(j=1, \cdots, h-1), v_{1}^{\prime}=r_{0} ;
$$

moreover, if $v_{i}(x) \in \mathfrak{A}_{1}$ and satisfy $(2.4)$ then $v_{i}(x) \in \mathfrak{A}_{1 ; 2},(i=1, \cdots, n)$, whenever $r_{j}(x) \in \Omega_{2},(j=0,1, \cdots, h-1)$.

If (2.4) is satisfied by $v_{i}(x) \in \mathfrak{A}_{1}$ then for $y \in \mathfrak{A}_{h}$ one has $\sum_{j=0}^{h} r_{j} y^{(j)}$ $=\left[\sum_{j=0}^{h-1} v_{j+1} y^{(j)}\right]^{\prime}$, and consequently $H_{k},(k \geqq h)$, is immediate. On the other hand, if for some integer $k \geqq h$ the condition $H_{k}$ is valid then by Theorem 2.1 there is a $P_{h-1}(x) \in \Re_{h-1}$ such that $\sum_{j=0}^{h}(-1)^{i} S_{j}\left(x ; r_{h-j}\right)=P_{h-1}(x)$, and $v_{j}(x)$ $=(-1)^{h-i} P_{h-1}^{(n-j)}(x)-\sum_{i=1}^{j}(-1)^{i} S_{i}\left(x ; r_{j-i}\right),(j=1, \cdots, h)$, are absolutely continuous functions satisfying (2.4). The last statement of the corollary is an immediate consequence of (2.4).

Let $\mathfrak{G}_{\mathfrak{k}}^{\odot}$ denote the class of functions $f(x) \in \mathfrak{C}_{k}$ such that $f(x) \equiv 0$ outside some subinterval $a_{1} \leqq x \leqq b_{1}$, where $a<a_{1}<b_{1}<b$, and the values of $a_{1}$ and $b_{1}$ are dependent on the particular function $f(x)$. The condition that the integral $I(y)$ of $(2.1)$ be zero for $y \in \mathfrak{C}_{\mathbf{k}}^{\odot}$ will be denoted by $H_{\mathbf{k}}^{\odot}$.

Corollary 2. For $k \geqq h$ the condition $H_{k}^{\odot}$ is equivalent to $H_{k}$.

Clearly $H_{k}$ implies $H_{k}^{\odot}$, since $\mathfrak{G}_{k}^{\odot} \subset \mathfrak{S}_{k}$. On the other hand, suppose that $H_{\boldsymbol{k}}^{\odot}$ holds and for a nondegenerate subinterval $\delta: a_{1} \leqq x \leqq b_{1}$ of $a<x<b$ let $\mathfrak{夭}_{k}(\delta)$ denote the set of functions $f(x) \in \mathfrak{夭}_{k}$ on $\delta$, and $\mathfrak{S}_{k}^{\circ}(\delta)$ the subclass of functions $f(x) \in \mathfrak{夭}_{k}(\delta)$ satisfying $f^{(\alpha)}\left(a_{1}\right)=0=f^{(\alpha)}\left(b_{1}\right),(\alpha=0,1, \cdots, k-1)$. If 
$y(x) \in \mathfrak{C}_{\mathbf{k}+1}^{\circ}(\delta)$ then $\eta(x)=y(x)$ on $\delta, \eta(x) \equiv 0$ elsewhere, is such that $\eta \in \mathfrak{F}_{\mathbf{k}}^{\odot}$, and consequently $H_{\boldsymbol{k}}^{\odot}$ implies that

$$
\int_{a_{1}}^{b_{1}}\left[\sum_{j=0}^{h} r_{j}(x) y^{(j)}\right] d x=0, \quad \text { for } y \in \mathfrak{夭}_{k+1}^{\circ}(\delta) .
$$

Theorem 2.1 then implies the existence of $P_{h-1}(x ; \delta) \in \mathfrak{B}_{h-1}$ such that $r(x)$ $\equiv \sum_{j=0}^{h}(-1)^{i} S_{j}\left(x ; r_{h-j}\right)=P_{h-1}(x ; \delta)$ on $\delta$. If $\delta_{1}, \delta_{2}$ are two such subintervals with $\delta_{1} \subset \delta_{2}$ it then follows that $P_{h-1}\left(x ; \delta_{2}\right)=P_{h-1}\left(x ; \delta_{1}\right)$ on $\delta_{1}$ and hence $P_{h-1}\left(x ; \delta_{2}\right) \equiv P_{h-1}\left(x ; \delta_{1}\right)$. Consequently $r(x)=P_{h-1}(x ; \delta)$ on $a b$, where $\delta$ is an arbitrary nondegenerate subinterval of $a<x<b$, and therefore $H_{k}$ holds in view of Theorem 2.1 .

For a second linear homogeneous differential expression

$$
M(z) \equiv \sum_{\nu=0}^{m} q_{\nu}(x) z^{(\nu)}, \text { with } q_{\nu}(x) \in \mathfrak{R}, \quad(\nu=0,1, \cdots, m),
$$

and $k \geqq \max (n, m)$, let $(L, M ; k)$ denote the condition that the functional

$$
(L(y), z)-(y, M(z))
$$

is zero for arbitrary $z \in \mathfrak{S}_{k}, y \in \mathfrak{C}_{k}^{\circ}$. The symbol $(L, M ; k)^{\circ}$ will denote the condition that (2.6) is zero for arbitrary $z \in \mathfrak{C}_{\mathbf{k}}^{\circ}, y \in \mathfrak{C}_{\mathbf{k}}^{\circ}$, and $(L, M ; k)^{\odot}$ the condition that (2.6) is zero for arbitrary $z \in \mathfrak{C}_{\mathbf{k}}^{\odot}, y \in \mathfrak{C}_{\mathfrak{k}}^{\odot}$.

THEOREM 2.2. If $L(y), M(z)$ are of the form (1.1) and (2.5), respectively, then for each $k \geqq \max (n, m)$ the conditions $(L, M ; k),(L, M ; k)^{\circ}$, and $(L, M ; k)^{\odot}$ are equivalent.

As $\mathfrak{C}_{k}^{\odot} \subset \mathfrak{C}_{k}^{\circ} \subset \mathfrak{C}_{k}$, clearly $(L, M ; k) \rightarrow(L, M ; k)^{\circ} \rightarrow(L, M ; k)^{\odot}$, and for the proof of the theorem it suffices to show that $(L, M ; k)^{\odot} \rightarrow(L, M ; k)$. Let $k$ be a fixed integer $k \geqq \max (n, m)$, and for brevity set $\{y, z\}=(L(y), z)-(y, M(z))$. For $0<\epsilon<(b-a) / 2$, denote by $\phi_{\epsilon}(x)$ a function of class $\mathfrak{E}_{k}$ such that $\phi_{\epsilon}(x) \equiv 1$ on $a+\epsilon \leqq x \leqq b-\epsilon$, and $\phi_{\epsilon}(x) \equiv 0$ on $a \leqq x \leqq a+\epsilon / 2$ and $b-\epsilon / 2 \leqq x \leqq b$. If $z \in \mathbb{E}_{k}$, $y \in \mathcal{C}_{k}^{\odot}$, then for $\epsilon$ a positive number so small that $y(x) \equiv 0$ on $a \leqq x \leqq a+\epsilon$ and $b-\epsilon \leqq x \leqq b$ we have $\{y, z\}=\left\{y, \phi_{\epsilon} z\right\}=0$ in case condition $(L, M ; k)^{\odot}$ holds. Now for a given $z \in \mathfrak{C}_{k}$ the functional $\{y, z\}$ is of the form (2.1), and in view of the above Corollary 2 it follows that for fixed $z \in \mathbb{C}_{k}$ the condition $\{y, z\}=0$ for arbitrary $y \in \mathfrak{C}_{\mathbf{k}}^{\odot}$ is equivalent to the condition that $\{y, z\}=0$ for arbitrary $y \in \mathfrak{C}_{\mathbf{k}}^{\circ}$. Consequently $(L, M ; k)^{\odot}$ implies $(L, M ; k)$ and the theorem is proved.

It is to be commented that with the aid of Theorem 2.1 and the above Corollary 2 one may show that each of the conditions of Theorem 2.2 is also equivalent to the condition that the expression (2.6) be zero for arbitrary $y \in \mathfrak{C}_{n}^{\odot}, z \in \mathfrak{C}_{m}^{\odot}$; a precise characterization of $L(y)$ and $M(z)$ is given in Corollary 2 to Theorem 3.2 . 
3. Characterization of the adjoint operator. In view of Corollary 1 to Theorem 2.1 the following general property of the adjoint operator is immediate.

THEOREM 3.1. The domain $\mathfrak{D}^{*}$ of the operator $T_{o}^{*}$ adjoint to $T_{o}$ is the totality of functions $z \in \mathfrak{R}$ for which $\bar{z} p_{\mu} \in \mathfrak{R},(\mu=0,1, \cdots, n)$, and there are functions $w_{j}=w_{j}(x ; z) \in \mathfrak{A}_{1},(j=1, \cdots, n)$, such that $w_{n}=z \bar{p}_{n}, w_{j+1}^{\prime}+w_{j}=z \bar{p}_{j}$, $(j=1, \cdots, n-1)$; moreover, if $z \in D^{*}$ then $T_{o}^{*} z=z \bar{p}_{o}-w_{1}^{\prime}(x ; z)$ and $w_{j} \in \mathfrak{A}_{1 ; 2}$, $(j=1, \cdots, n)$, whenever $T_{o}^{*} z$ and $z p_{j},(j=0,1, \cdots, n-1)$, belong to $\mathfrak{R}_{2}$.

From Theorem 3.1 it follows that in general the value of $T_{o}^{*} z$ is not given by a differential expression of the form (1.1), but by a "quasi-differential expression" in the sense of Bôcher [2]. The reader is referred to Halperin [4] for a discussion of general quasi-differential operators in a Hilbert space setting and under regularity assumptions corresponding to hypothesis $(\mathrm{H})$ of $\S 4$ below.

In particular, if $p_{\mu} \in \mathfrak{A}_{\mu},(\mu=0,1, \cdots, n)$, then $z p_{\mu} \in \mathfrak{A}_{\mu}$ for arbitrary $z \in \mathfrak{A}_{n}$, the domain $\mathfrak{D}^{*}$ of $T_{o}^{*}$ contains $\mathfrak{A}_{n}$, and $T_{o}^{*} z=\sum_{\mu=0}^{n}(-1)^{\mu}\left(z \bar{p}_{\mu}\right)^{(\mu)}$ for $z \in \mathfrak{A}_{n}$. Finally, if $p_{\mu} \in \mathfrak{A}_{\mu},(\mu=0,1, \cdots, n)$, and $p_{n}(x) \neq 0$ on $a b$, then $z \in \mathfrak{D}^{*}$ if and only if $z=w_{n} / p_{n}$, where $w_{i} \in \mathfrak{A}_{1}, \quad(i=1, \cdots, n)$, and $w_{j+1}^{\prime}+w_{j}$ $=\left(\bar{p}_{j} / \bar{p}_{n}\right) w_{n},(j=1, \cdots, n-1)$, from which it follows readily that $w_{n} \in \mathfrak{A}_{n}$, and consequently $w_{n} / \bar{p}_{n} \in \mathfrak{A}_{n}$. That is, if $p_{\mu} \in \mathfrak{A}_{\mu},(\mu=0,1, \cdots, n)$, and $p_{n}(x)$ $\neq 0$ on $a b$, then $\mathfrak{A}_{n}=\mathfrak{D}^{*}$ and $T_{o}^{*} z=\sum_{\mu=0}^{n}(-1)^{\mu}\left(z \bar{p}_{\mu}\right)^{(\mu)}$ for $z \in \mathfrak{A}_{n}$.

Throughout the following discussion the symbol $k_{j}$ will be used to denote $j / 2$ or $(j+1) / 2$ according as $j$ is even or odd; also, we shall employ $g_{\lambda}(x)$ for the particular polynomials $g_{o}(x) \equiv 1, g_{\lambda}(x) \equiv x^{r} / \lambda !,(\lambda=1,2, \cdots)$.

THEOREM 3.2. If $g_{\lambda}(x) \in \mathfrak{D}^{*},\left(\lambda=0,1, \cdots, k_{n}-1\right)$, then there exist functions $\pi_{j}(x) \in \mathfrak{A}_{k_{j}},(j=0,1, \cdots, n)$, such that

$$
L(y)=\sum_{j=0}^{n} \Lambda_{j}\left(y ; \pi_{j}\right), \quad \text { for } y \in \mathfrak{A}_{n} ;
$$

also, $\mathfrak{A}_{n} \subset \mathfrak{D}^{*}$ and

$$
T_{o}^{*} z=L^{\star}(z) \equiv \sum_{j=0}^{n} \bigwedge_{j}\left(z ;(-1)^{i} \bar{\pi}_{j}\right), \quad \text { for } z \in \mathfrak{A}_{n} .
$$

Moreover, $\pi_{j}(x) \in \mathfrak{A}_{k_{j ;} ;},(j=0,1, \cdots, n)$, if and only if $T_{o}^{*} g_{\lambda} \in \mathfrak{R}_{2},(\lambda=0,1, \cdots$, $\left.k_{n}-1\right)$, and $p_{j}(x) \in \Omega_{2},\left(j=0,1, \cdots, n-k_{n}\right)$.

The result of this theorem will be established by induction. For $n=1$ we have $L(y)=p_{1}(x) y^{\prime}+p_{o}(x) y, k_{1}=1$, and the hypothesis that $g_{o}(x) \equiv 1$ belongs to the corresponding set $\mathfrak{D}^{*}$ implies that $q_{o}(x) \equiv T_{o}^{*} g_{o} \in \mathcal{R}$ and

$$
\int_{a}^{b}\left[L(y)-\bar{q}_{o} y\right] d x=0, \quad \text { for } y \in \mathfrak{C}_{1}^{\circ} \text {. }
$$


From Theorem 2.1 for $h=1$ it follows that there exists a $\pi_{1}(x) \in \mathfrak{A}_{1}$ such that $\pi_{1}=p_{1}, \pi_{1}^{\prime}=p_{o}-\bar{q}_{o}$, and consequently $L(y)=\Lambda_{1}\left(y ; \pi_{1}\right)+\Lambda_{o}\left(y ; \pi_{o}\right)$, with $\pi_{o}$ $=p_{o}-\pi_{1}^{\prime} / 2$. If $z \in \mathfrak{A}_{1}$ then $\bar{z} p_{\mu} \in \mathfrak{R},(\mu=0,1), L^{\star}(z) \equiv \Lambda_{1}\left(z ;-\bar{\pi}_{1}\right)+\Lambda_{o}\left(z ; \bar{\pi}_{o}\right) \in \mathbb{R}$, and $(L(y), z)-\left(y, L^{\star}(z)\right)=0$ for $z \in \mathfrak{A}_{1}, y \in \mathfrak{C}_{1}^{\circ}$ in view of (1.3), so that $\mathfrak{A}_{1} \subset \mathfrak{D}^{*}$ and $\left(y, L^{\star}(z)-T_{o}^{*} z\right)=0$ for arbitrary $y \in \mathfrak{C}_{1}^{\circ}$. From the result of Theorem 2.1 for $h=0$ it then follows that $T_{o}^{*} z=L^{\star}(x)$ for $z \in \mathfrak{A}_{1}$. To establish for $n=1$ the last conclusion of the theorem it is to be noted that if $p_{o} \in \Omega_{2}$ and $q_{o} \equiv T_{o}^{*} g_{o} \in \Omega_{2}$ then the above defined function $\pi_{1}$ has $\pi_{1}^{\prime} \in \mathcal{R}_{2}$ and hence $\pi_{1} \in \mathfrak{P}_{1 ; 2}$, while $\pi_{o}=p_{o}-\pi_{1}^{\prime} / 2 \in \Omega_{2} \equiv \mathfrak{A}_{o ; 2}$. Conversely, if $\pi_{j} \in \mathfrak{A}_{k_{j ; 2}},(j=0,1)$, then from (3.1) and (3.2) it follows that $T_{o}^{*} z \in \mathfrak{R}_{2}$ for arbitrary $z \in \mathfrak{R}_{1 ; 2}$ and $p_{\mu} \in \mathfrak{R}_{2},(\mu=0,1)$.

Now suppose that the result of the theorem is valid for $n=m-1 \geqq 1$, and let $L(y)=\sum_{\mu=0}^{m} p_{\mu}(x) y^{(\mu)}(x)$ with $p_{\mu}(x) \in R,(\mu=0,1, \cdots, m)$, be such that $g_{\lambda}(x),\left(\lambda=0,1, \cdots, k_{m}-1\right)$, belongs to the corresponding $\mathfrak{D}^{*}$. If $f(x)$, $g(x) \in \mathfrak{A}_{m}$ it may be verified readily by induction that

$$
g f^{(\mu)}=\sum_{j=0}^{\mu}(-1)^{i} C_{\mu, j}\left[g^{(j)} f\right]^{(\mu-j)}, \quad(\mu=0,1, \cdots, m),
$$

where $C_{\mu, j}$ is the binomial coefficient $\mu ! /[j !(\mu-j) !]$. In particular, for $z, y \in \mathfrak{A}_{m}$,

$$
\bar{z} L(y)=\sum_{\sigma=0}^{m}(-1)^{\sigma} L_{\sigma}\left(\bar{z}^{(\sigma)} y\right),
$$

with

$$
L_{\sigma}(u)=\sum_{\mu=\sigma}^{m} C_{\mu, \sigma} p_{\mu}(x) u^{(\mu-\sigma)}, \quad(\sigma=0,1, \cdots, m) .
$$

Now the condition that $g_{\lambda}(x) \in D^{*},\left(\lambda=0,1, \cdots, k_{m}-1\right)$, implies that

$$
\left(y, T_{o}^{*} g_{\lambda}\right)=\left(L(y), g_{\lambda}\right)=\sum_{\sigma=0}^{\lambda}(-1)^{\sigma}\left(L_{\sigma}\left(g_{\lambda-\sigma} y\right), 1\right),\left(\lambda=0,1, \cdots, k_{m}-1\right)
$$

for arbitrary $y \in \mathfrak{C}_{m}^{\circ}$. If the integrable functions $q_{\lambda}(x),\left(\lambda=0,1, \cdots, k_{m}-1\right)$, are defined as

$$
q_{0}(x)=T_{o}^{*} g_{o}, \quad q_{\lambda}(x)=T_{o}^{*} g_{\lambda}-\sum_{j=0}^{\lambda-1} g_{\lambda-j}(x) q_{j}(x), \quad\left(j=1, \cdots, k_{m}-1\right),
$$

then in view of the fact that $g_{\lambda} y \in \mathfrak{C}_{m}^{\circ}$ whenever $y \in \mathfrak{C}_{m}^{\circ}$ it follows readily by induction that (3.5) implies

$$
I_{\sigma}(y) \equiv \int_{a}^{b}\left[L_{\sigma}(y)-(-1)^{\sigma} \bar{q}_{\sigma} y\right] d x \text { for } y \in \mathfrak{C}_{m}^{\circ},\left(\sigma=0,1, \cdots, k_{m}-1\right)
$$

As $L_{\sigma}(y)$ is of order at most $m-\sigma$, it follows from Theorem 2.1 that

$$
I_{\sigma}(u)=0 \text { for } u \in \mathfrak{A}_{m-\sigma}^{\circ}, \quad\left(\sigma=0,1, \cdots, k_{m}-1\right) .
$$

Now if $y \in \mathfrak{C}_{m}^{\circ}$ then $y^{(\sigma)} \in \mathfrak{C}_{m-\sigma}^{\circ},\left(\sigma=0,1, \cdots, k_{m}-1\right)$, and consequently (3.8) implies 


$$
I_{\sigma}\left(y^{(\sigma)}\right)=0 \text { for } y \in \mathfrak{C}_{m}^{\circ}, \quad\left(\sigma=0,1, \cdots, k_{m}-1\right) .
$$

For $j=0, \cdots, m$ the coefficient of $y^{(j)}$ in $L_{\sigma}\left(y^{(\sigma)}\right)$ is $C_{j, \sigma} p_{j}(x)$. Moreover, for $0 \leqq 2 \tau \leqq m$ the $(\tau+1) \times(\tau+1)$ matrix $\left\|C_{m-\beta, \alpha}\right\|,(\alpha, \beta=0,1, \cdots, \tau)$, is nonsingular. Indeed, if $\Delta_{m, \tau}$ denotes the determinant of this matrix then $\Delta_{m, \tau}$ $=(-1)^{\tau(\tau+1) / 2},(0 \leqq 2 \tau \leqq m ; m=0,1, \cdots)$, in view of the fact that $\Delta_{m, 0}=1$, $(m=0,1, \cdots)$, and the readily derivable relation $\Delta_{m, \tau}=(-1)^{\tau} \Delta_{m-1, \tau-1}$. Consequently, for $\tau=k_{m}-1$ there exist unique constants $e_{\alpha}$ such that $\sum_{\alpha=0}^{\tau} C_{m-\beta, \alpha} e_{\alpha}=\delta_{\beta, 0}, \quad(\beta=0,1, \cdots, \tau)$, and hence $\sum_{\alpha=0}^{\tau} e_{\alpha}\left[L_{\alpha}\left(y^{(\alpha)}\right)\right.$ $\left.-(-1)^{\alpha} \bar{q}_{\alpha} y^{(\alpha)}\right]$ is of the form $\sum_{j=0}^{m} r_{j}(x) y^{(j)}$ with $r_{m}(x)=p_{m}(x)$, and $r_{j}(x)=0$ for $m-k_{m}<j<m$. Indeed, from the expression for $e_{\alpha}$ in terms of the cofactors of $\left\|C_{m-\beta, \alpha}\right\|$ it follows that the coefficient of $y^{\left(m-k_{m}\right)}$ in $\sum_{\alpha=0}^{r} e_{\alpha} L_{\alpha}\left(y^{(\alpha)}\right)$ is equal to $(-1)^{\tau} p_{m-k_{m}}(x) \Delta_{m-1, \tau} / \Delta_{m, \tau}=(-1)^{\tau} p_{m-k_{m}}(x)$, and hence $r_{m-k_{m}}(x)$ $=(-1)^{\tau} p_{m-k_{m}}(x)$ if $m$ is even, $r_{m-k_{m}}(x)=(-1)^{\tau}\left[p_{m-k_{m}}(x)-\bar{q}_{m-k_{m}}(x)\right]$ $=(-1)^{\tau}\left[p_{m-k_{m}}(x)-\bar{q}_{k_{m}-1}(x)\right]$ if $m$ is odd. From Theorem 2.1 for $h=m$ it follows that there exists a function $\pi_{m} \in \mathfrak{A}_{k_{m}}$ such that $\pi_{m}=p_{m}$ and $\pi_{m} \in \mathfrak{A}_{k_{m}, 2}$ whenever $r_{m-k_{m}} \in \Omega_{2}$. Now the condition that $T_{o}^{*} g_{\lambda} \in \Omega_{2},\left(\lambda=0,1, \cdots, k_{m}-1\right)$, implies that $q_{k_{m}-1}(x) \in \Omega_{2}$ in view of (3.6), and from the value of $r_{m-k_{m}}$ derived above it follows that $\pi_{m} \in \mathfrak{A}_{k_{m} ;^{2}}$ whenever $p_{m-k_{m}} \in \mathfrak{R}_{2}$ and $T_{o}^{*} g_{\lambda} \in \mathfrak{R}_{2}$, $\left(\lambda=0,1, \cdots, k_{m}-1\right)$.

For $\pi_{m}$ determined as above, let $T_{1} y$ denote the operator with value $T_{1} y=L^{(1)}(y) \equiv L(y)-\Lambda_{m}\left(y ; \pi_{m}\right) \equiv \sum_{\nu=0}^{m-1} p_{\nu}^{(1)}(x) y^{(\nu)}$ on the domain $\mathfrak{E}_{m-1}^{\circ}$, and denote by $\mathfrak{D}_{1}^{*}$ the domain of the operator $T_{1}^{*} z$ adjoint to $T_{1} y$ in the sense defined in the introduction. As the order of the lowest derivative occurring in $\Lambda_{m}(z$; $\left.(-1)^{m} \bar{\pi}_{m}\right)$ is $k_{m-1}=m-k_{m}$, in view of (1.3) it follows that for $\lambda=0,1, \cdots$, $k_{m-1}-1$ we have $\left(L^{(1)}(y), g_{\lambda}\right)=\left(L(y), g_{\lambda}\right)-\left(\Lambda_{m}\left(y ; \pi_{m}\right), g_{\lambda}\right)=\left(y, T_{o}^{*} g_{\lambda}\right)-\left(y, \Lambda\left(g_{\lambda}\right.\right.$; $\left.\left.(-1)^{m} \bar{\pi}_{m}\right)\right)=\left(y, T_{o}^{*} g_{\lambda}\right)$ if $y \in \mathbb{C}_{m}^{\circ}$; as the order of $L^{(1)}(y)$ is at most $m-1$ it follows from Theorem 2.1 that $\left(L^{(1)}(y), g_{\lambda}\right)=\left(y, T_{o}^{*} g_{\lambda}\right)$ if $y \in \mathfrak{S}_{m-1}^{\circ}$, so that $g_{\lambda}(x) \in \mathfrak{D}_{1}^{*}$ and $T_{1}^{*} g_{\lambda}=T_{o}^{*} g_{\lambda}$ for $\lambda=0,1, \cdots, k_{m-1}-1$. The inductive hypothesis that the result of the theorem is true for $n=m-1$ when applied to the operator $T_{1} y$, together with the relation $L(y)=L^{(1)}(y)+\Lambda_{m}\left(y ; \pi_{m}\right)$, yields immediately the result that the conclusion of the first sentence of the theorem is true for $n=m$.

For the attainment by the same inductive argument that $\pi_{j} \in \mathfrak{A}_{\boldsymbol{k}_{j} ; 2}$, $(j=0,1, \cdots, m)$, whenever $T_{o}^{*} g_{\lambda} \in \Omega_{2},\left(\lambda=0,1, \cdots, k_{m}-1\right)$, and $p_{j}(x) \in \Omega_{2}$, $\left(j=0,1, \cdots, n-k_{n}\right)$, it remains to show that these conditions imply that $T_{1}^{*} g_{\lambda} \in \Omega_{2}, \quad\left(\lambda=0,1, \cdots, k_{m-1}-1\right)$, and $p_{i}^{(1)}(x) \in \Omega_{2}, \quad(i=0,1, \cdots, m-1$ $\left.-k_{m-1}\right)$. The first result is immediate in view of the above-mentioned fact that $T_{1}^{*} g_{\lambda}=T_{0}^{*} g_{\lambda},\left(\lambda=0,1, \cdots, k_{m-1}-1\right)$. The validity of the second result follows from the following comments: if $m$ is even then $k_{m-1}=k_{m}, m-1-k_{m-1}$ $=m-k_{m}-1$, and $p_{i}^{(1)}=p_{i},\left(i=0,1, \cdots, m-1-k_{m-1}\right)$; if $m$ is odd then $k_{m-1}=k_{m}-1, \quad \theta \equiv m-1-k_{m-1}=m-k_{m}, \quad p_{i}^{(1)}=p_{i}, \quad(i=0,1, \cdots, \theta-1), p_{\theta}^{(1)}$ $=p_{0}-\pi_{m}^{\left(k_{m}\right)} / 2$, while from the above established results it follows that the 
stated hypotheses imply that $\pi_{m} \in \mathfrak{A}_{k_{m} ; 2^{2}}$ Conversely, if $\pi_{j} \in \mathfrak{P}_{k_{j ;} ;},(j=0,1, \cdots$, $m$ ), then from (3.1) and (3.2) for $n=m$ it follows that $T_{o}^{*} z \in \mathfrak{R}_{2}$ for arbitrary $z \in \mathfrak{A}_{m ; 2}$ and $p_{\mu} \in \mathfrak{R}_{2},(\mu=0,1, \cdots, m)$.

For the explicit form of the bilinear concomitant $K_{n}(u, v ; p)$ $\equiv \sum_{i, j=1}^{n} u^{(i-1)} K_{i j ; n}(x ; p) v^{(j-1)}$ of $(1.3)$ the reader is referred, for example, to Kamke [8, p. 77]. The following readily verifiable properties will be employed here: for $n=2 r$ and $n=2 r-1$ each element $K_{i j ; n}$ is a linear combination of $p, p^{\prime}, \cdots, p^{(r-1)}$ with real coefficients, and thus $K_{i j i n} \in \mathfrak{A}_{1}$ for $p \in \mathfrak{A}_{r}$ and $K_{i j ; n} \in \mathfrak{A}_{1 ; 2}$ whenever $p \in \mathfrak{A}_{r ; 2} ; K_{i j ; n}=(-1)^{n-1} K_{j i ; n},(i, j=1, \cdots, n) ; K_{i j ; n}=0$ if $i+j>n+1 ; K_{i j, n}=(-1)^{i-1} p(x)$ if $i+j=n+1$. From these properties of the bilinear concomitant in (1.4), and the result of Theorem 3.2, the following conclusion is immediate with $\sum_{i, j=1}^{n} \bar{z}^{(i-1)} K_{i j}(x) y^{(j-1)} \equiv \sum_{\sigma=1}^{n} K_{\sigma}\left(\bar{z}, y ; \pi_{\sigma}\right)$, and $\pi_{\sigma}(x)$ as in (3.1).

Corollary 1. If $x^{\lambda} / \lambda ! \in \mathfrak{D}^{*},\left(\lambda=0,1, \cdots, k_{n}-1\right)$, then the differential expression $L^{\star}(z)$ defined in Theorem 3.2 satisfies with $L(y)$ the relation $\left({ }^{1}\right)$

$$
\bar{z} L(y)-y \bar{L}^{\star}(z)=\left[\sum_{i, j=1}^{n} \bar{z}^{(i-1)} K_{i j}(x) y^{(j-1)}\right]^{\prime} \quad \text { for } y, z \in \mathfrak{A}_{n},
$$

where $K_{i j} \in \mathfrak{A}_{1},(i, j=1, \cdots, n), K_{i j} \equiv 0$ for $i+j>n+1$, and $K_{i j}=(-1)^{i-1} \pi_{n}(x)$ or $i+j=n+1$, with $\pi_{n}(x)$ as in (3.1); moreover, $K_{i j} \in \mathfrak{A}_{1 ; 2},(i, j=1, \cdots, n)$, whenever $T_{o}^{*} g_{\lambda} \in \Omega_{2},\left(\lambda=0,1, \cdots, k_{n}-1\right)$, and $p_{j}(x) \in \Omega_{2},(j=0,1, \cdots$, $\left.n-k_{n}\right)$.

Corollary 2. If $L(y)$ and $M(z)$ are of the form (1.1) and (2.5), respectively, with coefficients belonging to $\&$, then for a given integer $k \geqq \max (n, m)$ a necessary and sufficient condition for the validity of the relation $(L, M ; k)^{\odot}$ is that $L(y)$ is of the form (3.1) with $\pi_{j}(x) \in \mathfrak{A}_{k_{j}},(j=1, \cdots, n)$, and $M(z)=L^{\star}(z)$. Moreover, if $(L, M ; k)^{\odot}$ holds for some $k \geqq \max (n, m)$ then $\pi_{j}(x) \in \mathfrak{A}_{k_{j} ; 2},(j=0,1, \cdots, n)$, if and only if $g_{j}(x) \in \Omega_{2},\left(j=0,1, \cdots, k_{n}-1\right)$, and $p_{j}(x) \in \mathfrak{R}_{2},(j=0,1, \cdots$, $n-k_{n}$ ).

For $k \geqq \max (n, m)$ the relation $(L, M ; k) \odot$ implies the relation $(L, M ; k)$ by Theorem 2.2, so that for each $z \in \mathfrak{E}_{k}$ we have $\{y, z\} \equiv(L(y), z)-(y, M(z))$ $=0$ for $y \in \mathfrak{C}_{k}^{\circ}$; in particular, $\{y, z\}$ is of the form (2.1) with $h=n$, and from Theorem 2.1 it follows that $\{y, z\}=0$ for $z \in \mathfrak{C}_{k}, y \in \mathfrak{S}_{n}^{\circ}$. Consequently $\mathfrak{C}_{k} \subset \mathfrak{D}^{*}$, and $\left(y, T_{0}^{*} z-M(z)\right)=0$ for $y \in \mathfrak{C}_{n}^{\circ}, z \in \mathfrak{C}_{k}$, so that $T_{o}^{*} z=M(z)$ for $z \in \mathfrak{C}_{k}$. Therefore $g_{\lambda}(x) \equiv x^{\lambda} / \lambda ! \in \mathfrak{D}^{*},(\lambda=0,1, \cdots)$, and Theorem 3.2 implies that $L(y)$ is of the form (3.1) with $\pi_{j}(x) \in \mathfrak{A}_{k_{j}}$ and $T_{o}^{*} z=L^{\star}(z)$ for $z \in \mathfrak{A}_{n,}$, so that $M(z)=L^{\star}(z)$. Conversely, if $L(y)$ is of the form (3.1) and $M(z)=L^{\star}(z)$, then (3.9) implies the relation $(L, M ; k)^{\odot}$ for $k \geqq n$. Finally, if $(L, M ; k)^{\odot}$ holds for some $k \geqq \max (n, m)$, then $T_{o}^{*} g_{\lambda}=M\left(g_{\lambda}\right)=\sum_{\nu=0}^{\lambda} q_{\nu}(x) g_{\lambda-\nu}(x)$,

(1) In (3.9) the symbol $\bar{L}^{\star}(z)$ denotes the complex conjugate of $L^{\star}(z)$; in the following pages the symbols $\bar{M}(z)$ and $\bar{L}(z)$ will be used with similar meanings. 
$(\lambda=0,1, \cdots, n)$, and the final conclusion of the corollary is an immediate consequence of the corresponding conclusion of Theorem 3.2.

4. Additional results under suitable regularity assumptions. In the preceding discussion of adjoint operators no assumption was made on the nonvanishing of the leading coefficient $p_{n}(x)$ of (1.1). In particular, no use was made of the existence or character of solutions of the associated linear homogeneous differential equations. The results of the present section are for the case when the following hypothesis is satisfied:

(H) $p_{\mu}(x) \in \mathcal{R},(\mu=0,1, \cdots, n)$, and there is an $\epsilon_{o}>0$ such that $\left|p_{n}(x)\right|$ $\geqq \epsilon_{o}$ a.e. on $a b$.

If $(\mathrm{H})$ holds then for given $f(x) \in \mathbb{R}$ and constants $\eta_{i},(i=1, \cdots, n)$, wellknown existence theorems imply that there is a unique $y \in \mathfrak{A}_{n}$ such that

$$
L(y)=f(x), \quad y^{(i-1)}\left(x_{0}\right)=\eta_{i}, \quad(i=1, \cdots, n),
$$

where $x_{o}$ is a fixed value on $a b$; such a function is termed a solution of (4.1).

In case $(H)$ holds and $g_{\lambda}(x) \equiv x^{\lambda} / \lambda ! \in \mathfrak{D}^{*},\left(\lambda=0,1, \cdots, k_{n}-1\right)$, then the function $\pi_{n}(x)$ of Theorem 3.2 is such that either $\pi_{n} \geqq \epsilon_{o}$ or $\pi_{n} \leqq-\epsilon_{o}$ throughout $a b$. As the adjoint expression $L^{\star}(z)=\sum_{\mu=0}^{n} q_{\mu}(x) z^{(u)}$ given by (3.2) has $q_{n}=(-1)^{n} \bar{\pi}_{n}$, there is also a unique solution of the corresponding system

$$
L^{\star}(z)=f(x), \quad z^{(i-1)}\left(x_{o}\right)=\eta_{i},
$$

$$
(i=1, \cdots, n) \text {. }
$$

Since the condition that $\pi_{n}$ does not vanish on $a b$ implies that the matrix $\left\|K_{i j}(x)\right\|$ of (3.9) is nonsingular, it follows from (3.9) by classical methods that the system

$$
L(y)=f(x), \quad y^{(i-1)}(a)=0=y^{(i-1)}(b),
$$$$
(i=1, \cdots, n),
$$

with $f(x) \in \mathbb{R}$ has a solution if and only if $(f, z)=0$ for arbitrary solutions $z$ of $L^{\star}(z)=0$.

It is to be remarked that under hypothesis $(\mathrm{H})$ a solution $y(x)$ of $(4.1)$ is such that $y(x) \in \mathfrak{A}_{n ; 2}$ in case $f(x) \in \mathfrak{R}_{2}$ and $p_{j}(x) \in \mathfrak{R}_{2},(j=0,1, \cdots, n-1)$. If (H) holds, $g_{\lambda}(x) \equiv x^{\lambda} / \lambda ! \in \mathfrak{D}^{*}$ with $T_{o}^{*} g_{\lambda} \in \Omega_{2}, \quad\left(\lambda=0,1, \cdots, k_{n}-1\right)$, and $p_{j}(x) \in \mathfrak{R}_{2},\left(j=0,1, \cdots, n-k_{n}\right)$, then by Theorem 3.2 we have that $\pi_{j}(x)$ $\in \mathfrak{A}_{k_{j} ; 2},(j=0,1, \cdots, n)$. In this case all coefficients of $L(y)$ and $L^{\star}(z)$ belong to $\mathfrak{R}_{2}$, and for a given $x_{o}$ on $a b$ all solutions of $(4.1)$ and $\left(4.1^{\prime}\right)$ belong to $\mathfrak{A}_{n ; 2}$ whenever $f(x) \in \mathfrak{R}_{2}$.

THEOREM 4.1. If hypothesis $(\mathrm{H})$ holds and $x^{\lambda} / \lambda ! \in D^{*},\left(\lambda=0,1, \cdots, k_{n}-1\right)$, then $\mathfrak{D}^{*}=\mathfrak{A}_{n}$.

To establish this theorem, let $z_{\alpha}(x),(\alpha=1, \cdots, n)$, be linearly independent solutions of $L^{\star}(z)=0$ which are orthonormal in the sense that $\left(z_{\alpha}, z_{\beta}\right)$ $=\delta_{\alpha \beta},(\alpha, \beta=1, \cdots, n)$. Now if $z_{o} \in \mathfrak{D}^{*}$, and $w_{o}(x)$ is a particular solution of $L^{\star}\left(w_{o}\right)=T_{o}^{*} z_{o}$, then $w(x)=w_{o}(x)+\sum_{\alpha=1}^{n}\left(z_{o}-w_{o}, z_{\alpha}\right) z_{\alpha}(x)$ is the solution of $L^{\star}(w)=T_{o}^{*} z_{o}$ such that $\left(z_{o}-w, z\right)=0$ for arbitrary solutions $z(x)$ of $L^{\star}(z)=0$. 
In view of (3.9) and the definition of $T_{o}^{*} z_{o}$ it follows that $\left(L(y), z_{o}\right)=\left(y, T_{o}^{*} z_{o}\right)$ $=\left(y, L^{\star}(w)\right)=(L(y), w)$, and consequently $\left(L(y), z_{o}-w\right)=0$, for arbitrary $y \in \mathfrak{C}_{n}^{\circ}$. By Theorem 2.1 it then follows that $\left(\bar{z}_{o}-\bar{w}\right) p_{n}$ is equivalent to an absolutely continuous function, and $\left(L(y), z_{o}-w\right)=0$ for arbitrary $y \in \mathfrak{H}_{n}^{\circ}$. Finally, since $\left(z_{o}-w, z\right)=0$ for arbitrary solutions of $L^{\star}(z)=0$, the above statement on the solvability of (4.2) implies that there is a $y \in \mathfrak{H}_{n}^{\circ}$ such that $L(y)=z_{o}-w$, and hence $\left(z_{o}-w, z_{o}-w\right)=0$ and $z_{o}=w \in \mathfrak{A}_{n}$.

The following theorem presents an extension of a result of Hamburger [5] for the Sturm-Liouville case; this latter case will be considered specifically in the next section.

THEOREM 4.2. If $L(y)$ satisfies (H) and $M(z)=\sum_{\nu=0}^{n} q_{\nu}(x) z^{(\nu)}$, with $q_{\nu}(x) \in \mathcal{R},(\nu=0,1, \cdots, n)$, then a necessary and sufficient condition that

$$
(L(y), z)=(y, M(z)), \quad \text { for } z \in \mathfrak{G}_{n}, y \in \mathfrak{C}_{n}^{\circ},
$$

is: (a) $q_{n}(x)=(-1)^{n} \bar{p}_{n}(x)$; (b) for linearly independent solutions $y_{j}(x)$ and $z_{j}(x),(j=1, \cdots, n)$, of $L(y)=0$ and $M(z)=0$, respectively, there exists a corresponding constant matrix $\Delta \equiv\left\|\Delta_{i j}\right\|,(i, j=1, \cdots, n)$, such that

$$
\sum_{i, j=1}^{n}\left[y_{i}^{(\alpha-1)}(x) \Delta_{i j} \bar{z}_{j}(x)\right]=\delta_{n \alpha} / p_{n}(x), \quad(\alpha=1, \cdots, n) .
$$

In particular, $(L(y), z)=(y, M(z))$ for $z \in \mathfrak{A}_{n}, y \in \mathfrak{A}_{n}^{\circ}$ whenever (4.3) holds.

In view of Theorem 2.2 and Corollary 2 of Theorem 3.2 condition (4.3) is equivalent to $M(z)=L^{\star}(z)$, so that (4.3) implies immediately conclusion (a). If $y=y_{j}(x)$ and $z=z_{j}(x),(j=1, \cdots, n)$, are linearly independent solutions of $L(y)=0$ and $L^{\star}(z)=0$, respectively, then the Wronskian matrices $Y(x)$ $\equiv\left\|y_{j}^{(i-1)}(x)\right\|$ and $Z(x) \equiv\left\|z_{j}^{(i-1)}(x)\right\|,(i, j=1, \cdots, n)$, are nonsingular on $a b$ and from (3.9) it follows that there is a constant $n \times n$ matrix $D$ such that $Z^{*}(x) K(x) Y(x) \equiv D$. In the preceding matrix identity, as well as in subsequent occurrences, the exponent ${ }^{*}$ on the symbol for a matrix denotes the conjugate transpose matrix. Since hypothesis $(\mathrm{H})$ implies that $K(x)$ is nonsingular the matrix $D$ is nonsingular, and $\Delta=D^{-1}$ is such that $Y(x) \Delta Z^{*}(x)$ is the reciprocal of $K(x)$. The relations $K_{i j}(x) \equiv 0$ for $i+j>n+1$, and $K_{i j}(x)$ $=(-1)^{i-1} \pi_{n}(x)$ for $i+j=n+1$, imply

$$
\begin{aligned}
\sum_{i, j=1}^{n}\left[y_{i}^{(\alpha-1)}(x) \Delta_{i j} \bar{z}_{j}^{(\beta-1)}(x)\right] & =0, \\
\sum_{i, j=1}^{n}\left[y_{i}^{(n-\beta)}(x) \Delta_{i} \bar{z}_{j}^{(\beta-1)}(x)\right] & =(-1)^{\beta-1} / p_{n}(x), \\
(\alpha & =1, \cdots, n-\beta ; \beta=1, \cdots, n),
\end{aligned}
$$

which provide relations (4.4) for $\beta=1, \alpha=1, \cdots, n-1$. In connection with statement (b) of the theorem it is to be commented that this condition is 
clearly equivalent to the validity of (4.4) for any one particular set of linearly independent solutions $y_{j}(x), z_{j}(x)$ with a corresponding constant matrix $\Delta$.

Now suppose that (H) holds for (1.1), and conditions (a) and (b) are satisfied by $L(y)$ and $M(z)=\sum_{\nu=0}^{n} q_{\nu}(x) z^{(\nu)}$, with $q_{\nu}(x) \in \mathfrak{R},(\nu=0,1, \cdots, n)$. It follows readily that relations (4.4) imply the extended set of conditions (4.5); indeed, if for a given $\gamma$ satisfying $1 \leqq \gamma \leqq n$ the $n-\gamma$ conditions of (4.5) specified by $\beta=\gamma, \alpha=1, \cdots, n-\gamma$ hold, then upon differentiation of the first $n-\gamma-1$ of these conditions one obtains the conditions of (4.5) specified by $\beta=\gamma+1$. Now conditions (4.5) imply that $H(x)=Y(x) \Delta Z^{*}(x)$ has $H_{i j}(x) \equiv 0$ for $i+j<n+1$, and $H_{i j}(x)=(-1)^{j-1} / p_{n}(x)$ for $i+j=n+1$. In particular, $H(x)$ is nonsingular a.e. on $a b$ and thus a matrix $\Delta$ satisfying (4.4) is nonsingular. Now for $y \in \mathbb{C}_{n}$ the function $r(x)=L(y) \in \mathbb{R}$, and in view of (4.4) it follows by the method of variation of parameters that $y(x)=y_{j}(x) h_{j}(x)$, where $h_{i}(x) \in \mathfrak{A}_{1}$ and $h_{i}^{\prime}(x)=\Delta_{i j} \bar{z}_{j}(x) r(x)$; for simplicity of notation, in the expressions for $y(x)$ and $h_{i}^{\prime}(x)$, and also in subsequent expressions of this paragraph, we employ the tensor analysis convention so that repetition of subscripts $i$ and $j$ in a given expression denotes summation over the range $1, \cdots, n$. Indeed, if $\eta_{i}$ and $h_{i}(x)$ are determined by $y^{(i-1)}(a)=y_{j}^{(i-1)}(a) \eta_{j}$, $h_{i}(x)=\eta_{i}+\Delta_{i j} \int_{a}^{x} \bar{z}_{j}(t) r(t) d t,(i=1, \cdots, n)$, then (4.4) imply $y_{j}^{(i-1)}(x) h_{j}^{\prime}(x)=0$, $(i=1, \cdots, n-1), p_{n}(x) y_{j}^{(n-1)}(x) h_{j}^{\prime}(x)=r(x)$, and hence $u(x) \equiv y_{j}(x) h_{j}(x) \in \mathfrak{A}_{n}$, $u^{(i-1)}(x) \equiv y_{j}^{(i-1)}(x) h_{j}(x), u^{(i-1)}(a)=y^{(i-1)}(a),(i=1, \cdots, n)$, and $L(u)=r(x)$, so that $u(x) \equiv y(x)$. Correspondingly, if $z \in \mathfrak{S}_{n}$ then $s(x) \equiv M(z) \in \mathfrak{R}$, and from the $n$ relations of (4.5) given by $\alpha=1, \beta=1, \cdots, n$, it follows that $z(x)$ $=z_{j}(x) g_{j}(x)$, where $g_{j}(x) \in \mathfrak{A}_{1}$ and $g_{j}^{\prime}(x)=-\bar{\Delta}_{i j} \bar{y}_{i}(x) s(x)$. If $D$ is defined as the reciprocal of the nonsingular matrix $\Delta$ then $\bar{z} L(y)-y \bar{M}(z)=\bar{g}_{j} \bar{z}_{j} r-\bar{s} y_{i} h_{i}$ $=\bar{g}_{j} D_{j i} h_{i}^{\prime}+\bar{g}_{j}^{\prime} D_{j i} h_{i}=\left[\bar{g}_{j} D_{j i} h_{i}\right]^{\prime}=\left[\sum_{i, j=1}^{n} \bar{z}^{(i-1)}(x) K_{i j}(x) y^{(j-1)}(x)\right]^{\prime}, \quad$ where $K(x)=Z^{*-1}(x) D Y^{-1}(x)$, and therefore $(L(y), z)=(y, M(z))$ if $z \in \mathfrak{C}_{n}, y \in \mathfrak{C}_{n}^{\circ}$. Consequently from Corollary 2 of Theorem 3.2 it follows that $L(y)$ has the form (3.1), $M(z)=L^{\star}(z)$, and the equality in (4.3) holds for $z \in \mathfrak{A}_{n}, y \in \mathfrak{A}_{n}^{\circ}$.

5. Sturm-Liouville operators. A formal differential operator (1.1) with $p_{\mu}(x) \in \mathfrak{R}$ will be said to be a Sturm-Liouville operator (SL-operator) if

$$
(L(y), z)=(y, L(z)), \text { for arbitrary } z \in \mathfrak{S}_{n}, y \in \mathfrak{夭}_{n}^{\circ} .
$$

This definition is a slight modification of that of Hamburger [5], who introduced this terminology for the case when $p_{\mu} \in \mathfrak{R}_{2},(\mu=0,1, \cdots, n)$, the condition of hypothesis $(\mathrm{H})$ holds, and the equality of (5.1) holds for arbitrary $z \in \mathfrak{A}_{n ; 2}, y \in \mathfrak{A}_{n ; 2}^{\circ}$. In view of Theorem 2.2 condition (5.1) is equivalent to the condition that $(L(y), z)=(y, L(z))$ for arbitrary $z, y \in \mathfrak{S}_{n}^{\circ}$, or indeed for arbitrary $z, y \in \mathfrak{G}_{n}^{\odot}$. The following criterion is a ready consequence of Corollary 2 to Theorem 3.2.

THEOREM 5.1. The formal differential operator $L(y)$ is an $S L$-operator if and 
only if $L(y)=\sum_{j=0}^{n} \Lambda_{j}\left(y: \pi_{j}\right)$ where $\pi_{j} \in \mathfrak{A}_{k_{j}}$ and $\pi_{j}=(-1)^{i} \bar{\pi}_{j},(j=0,1, \cdots, n)$; moreover, $\pi_{j} \in \mathfrak{A}_{k_{j} ; 2}$ if and only if $p_{j}(x) \in \mathfrak{R}_{2},\left(j=0,1, \cdots, n-k_{n}\right)$.

The following result is an immediate consequence of Theorem 4.2.

THEOREM 5.2. If $L(y)$ satisfies hypothesis $(\mathrm{H})$ then $L(y)$ is an $S L$-operator if and only if the following conditions hold: (a) $p_{n}(x)=(-1)^{n} \bar{p}_{n}(x)$; (b) for linearly independent solutions $y_{j}(x),(j=1, \cdots, n)$, of $L(y)=0$ there exists a corresponding constant matrix $\Delta \equiv\left\|\Delta_{i j}\right\|,(i, j=1, \cdots, n)$, such that

$$
\sum_{i, j=1}^{n}\left[y_{i}^{(\alpha-1)}(x) \Delta_{i j} \bar{y}_{j}(x)\right]=\hat{o}_{n \alpha} / p_{n}(x) \quad(\alpha=1, \cdots, n) .
$$

The criterion of the following theorem is that of Hamburger [5], with the modification of terminology mentioned at the beginning of this section.

THEOREM 5.3. If $L(y)$ satisfies hypothesis $(\mathrm{H})$ then a necessary and sufficien ${ }^{t}$ condition that $L(y)$ be an $S L$-operator is that for linearly independent solutions $y_{j}(x),(j=1, \cdots, n)$, of $L(y)=0$ there exists a corresponding constant matrix $\Delta \equiv\left\|\Delta_{i j}\right\|,(i, j=1, \cdots, n)$, that is skew-Hermitian and such that the relations (5.2) hold.

If $L(y)$ is an SL-operator then in view of Corollary 1 of Theorem 3.2 there is a matrix $K(x) \equiv\left\|K_{i j}(x)\right\|$ with absolutely continuous elements satisfying $K_{i j} \equiv 0$ for $i+j>n+1, K_{i j}=(-1)^{i-1} \pi_{n}(x)$ for $i+j=n+1$, such that

$$
\bar{z} L(y)-y \bar{L}(z)=\left[\sum_{i, j=1}^{n} \bar{z}^{(i-1)} K_{i j}(x) y^{(j-1)}\right]^{\prime} \quad \text { for } y, z \in \mathfrak{A}_{n} .
$$

It follows readily that $\sum_{i, j=1}^{n}\left[\bar{z}^{(i-1)}\left(K_{i j}+\bar{K}_{j i}\right) y^{(j-1)}\right]$ is a constant for arbitrary $y, z \in \mathfrak{P}_{n}$ and consequently that $K(x) \equiv-K^{*}(x)$. As in the proof of the first part of Theorem 4.2 hypothesis $(\mathrm{H})$ implies that $K(x)$ is nonsingular and (5.2) holds for $\Delta=D^{-1}$, where $D$ is the constant $n \times n$ matrix such that a given set $y_{j}(x),(j=1, \cdots, n)$, of linearly independent solutions of $L(y)=0$ with Wronskian matrix $Y(x) \equiv\left\|_{y_{j}^{(i-1)}}(x)\right\|$ satisfies $Y^{*}(x) K(x) Y(x) \equiv D$. The skewHermitian character of $K(x)$ implies that $\Delta=-\Delta^{*}$ and consequently the necessity of the stated condition is established.

On the other hand, if $\Delta=-\Delta^{*}$ and (5.2) holds for a set $y_{j}(x),(j=1, \cdots, n)$, of linearly independent solutions of $L(y)=0$, then as in the proof of the second part of Theorem 4.2 it follows that $H(x)=Y(x) \Delta Y^{*}(x)$ has $H_{i j}(x) \equiv 0$ for $i+j<n+1$ and $H_{i j}(x)=(-1)^{j-1} / p_{n}(x)$ for $i+j=n+1$. Then the condition $\Delta=-\Delta^{*}$ implies that $H(x) \equiv-H^{*}(x)$, and, in particular, that $p_{n}(x)$ $=(-1)^{n} \bar{p}_{n}(x)$. The conclusion that $L(y)$ is an SL-operator then follows from Theorem 5.2.

6. Extensions of the operator $T_{o}$. It will be supposed throughout this section that $L(y)$ is of the form (1.1) with $p_{\mu} \in \mathcal{R},(\mu=0,1, \cdots, n)$, and $x^{\lambda} / \lambda ! \in \mathfrak{D}^{*}$, 
$\left(\lambda=0,1, \cdots, k_{n}-1\right)$, so that $L(y)$ is of the form (3.1). For $\mathfrak{D}_{s}$ a linear subspace of $\mathfrak{A}_{n}$ containing $\mathfrak{A}_{n}^{\circ}$, let $T_{s}$ denote the operator with domain $\mathfrak{D}_{s}$ and value $T_{s} y=L(y)$ for $y \in \mathfrak{D}_{s}$. If $\mathfrak{D}_{s}^{*}$ is the totality of functions $z(x) \in \mathbb{R}$ for which $\bar{z} p_{\mu} \in R,(\mu=0,1, \cdots, n)$, and there exists a corresponding $f_{z}(x) \in \mathbb{R}$ such that $(L(y), z)=\left(y, f_{z}\right)$ for all $y \in \mathfrak{D}_{s}$ then the adjoint of $T_{s}$ is the operator $T_{s}^{*}$ with domain $\mathfrak{D}_{s}^{*}$ and value $T_{s}^{*} z=f_{z}$. If the linear subspace $\mathfrak{B}_{s}$ of complex $2 n$ dimensional space composed of the end-values $\left(y^{(j-1)}(a), y^{(j-1)}(b)\right)$, $(j=1, \cdots, n)$, for $y \in \mathfrak{D}_{s}$ has dimension $2 n-r$, and $M \equiv\left\|M_{\sigma j}\right\|, N \equiv\left\|N_{\sigma j}\right\|$, $(\sigma=1, \cdots, r ; j=1, \cdots, m)$, are such that $\mathfrak{B}_{s}$ is characterized by

$$
s_{\sigma}(y) \equiv \sum_{j=1}^{n}\left[M_{\sigma j} y^{(j-1)}(a)+N_{\sigma j} y^{(j-1)}(b)\right]=0, \quad(\sigma=1, \cdots, r),
$$

then the $r \times 2 n$ matrix $\left\|M_{\sigma j} N_{\sigma j}\right\|$ is of rank $r$ and $\mathfrak{D}_{s}$ is the totality of functions $y \in \mathfrak{A}_{n}$ satisfying (6.1). If $P \equiv\left\|P_{\tau j}\right\|_{1}, Q \equiv\left\|Q_{\tau j}\right\|,(\tau=1, \cdots, 2 n-r ; j=1, \cdots$, $n)$, are such that the $(2 n-r) \times 2 n$ matrix $\left\|P_{r j} Q_{r j}\right\|$ is of rank $2 n-r$ and $M P^{*}-N Q^{*}=0$, then in view of Theorem 3.2 and its Corollary 1 the domain $\mathfrak{D}_{\mathbf{s}}^{*}$ of the adjoint contains all $z \in \mathfrak{A}_{n}$ satisfying

$$
\sum_{i, j=1}^{n}\left[P_{\tau j} \bar{K}_{i j}(a) z^{(i-1)}(a)+Q_{\tau j} \bar{K}_{i j}(b) z^{(i-1)}(b)\right]=0, \quad(\tau=1, \cdots, 2 n-r),
$$

and $T_{s}^{*} z=L^{\star}(z)$ for such $z$.

For the remainder of this section it will be supposed that the hypothesis $(\mathrm{H})$ holds for (1.1). Then $\pi_{n}(x)$ does not vanish on $a b, K(x)$ is nonsingular, and in view of Theorem 4.1 and the fact that $\mathfrak{D}_{s}^{*} \subset \mathfrak{D}^{*}$ it follows that if $w \in \mathfrak{D}^{*}$ there is a $z \in \mathfrak{A}_{n}$ satisfying (6.2) such that $z=w$. It is to be remarked that the conditions (6.2) on the end-values $\left(z^{(i-1)}(a), z^{(i-1)}(b)\right)$ are equivalent to

$$
t_{\tau}(z) \equiv \sum_{j=1}^{n}\left[R_{\tau j} z^{(j-1)}(a)+S_{\tau j} z^{(j-1)}(b)\right]=0, \quad(\tau=1, \cdots, 2 n-r),
$$

if and only if the $(2 n-r) \times 2 n$ matrix $\left\|R_{\tau j} S_{r j}\right\|$ is of rank $2 n-r$ and

$$
M K^{-1}(a) R^{*}-N K^{-1}(b) S^{*}=0 .
$$

The consideration of adjoint systems where $r \neq n$ dates from Bôcher [2]. In Chapter 11 of [3] Coddington and Levinson treat in detail the problem of such systems for $L(y)=0$ under the assumption that $p_{\mu} \in \mathfrak{E}_{\mu},(\mu=0,1, \cdots, n)$, and state explicitly the criterion for adjointness in the form (6.4). A discussion equivalent to that of Coddington and Levinson, but one that seems simpler in detail to the author, is afforded by a procedure similar to that of Bliss $[1, \S 1]$, since $(6.3)$ is clearly equivalent to the condition imposed on $\zeta_{j}(a)=\sum_{i=1}^{n} z^{(i-1)}(a) \bar{K}_{i j}(a), \quad \zeta_{j}(b)=\sum_{i=1}^{n} z^{(i-1)}(b) \bar{K}_{i j}(b)$ by the restriction that $\sum_{j=1}^{n}\left[\bar{\zeta}_{j}(a) y^{(j-1)}(a)-\bar{\zeta}_{j}(b) y^{(j-1)}(b)\right]=0$ for all $y^{(i-1)}(a), y^{(i-1)}(b)$ satisfying (6.1). 
If $y_{j}(x) \in \mathfrak{A}_{n},(j=1, \cdots, n)$, and $Y(x)=\left\|y_{j}^{(i-1)}(x)\right\|$, the symbols $s(Y)$, $s^{1}(Y)$ are employed for the $r \times n$ matrices $s(Y)=M Y(a)+N Y(b), s^{1}(y)$ $=M Y(a)-N Y(b)$. Correspondingly, if $z_{j}(x) \in \mathfrak{A}_{n}, \quad(j=1, \cdots, n)$, we set $Z(x)=\left\|z_{j}^{(i-1)}(x)\right\|$ and define the $(2 n-r) \times n$ matrices $t(Z), t^{1}(Z)$ as $t(Z)$ $=R Z(a)+S Z(b), t^{1}(Z)=R Z(a)-S Z(b)$.

Theorem 6.1. Suppose that $L(y)$ satisfies $(\mathrm{H})$, and $T_{s}$ is the operator with $T_{s} y=L(y)$ on the domain $\mathfrak{D}_{s}$ of functions $y \in \mathfrak{H}_{n}$ satisfying (6.1), where the $r \times 2 n$ matrix $\left\|M_{\sigma j} N_{\sigma j}\right\|$ is of rank $r$. If $y_{j}(x), z_{j}(x),(j=1, \cdots, n)$, are linearly independent solutions of $L(y)=0, L^{\star}(z)=0$, respectively, and $\Delta$ is the corresponding constant matrix satisfying (4.4), then (6.3) defines the manifold of end-values $z^{(i-1)}(a), z^{(i-1)}(b),(i=1, \cdots, n)$, of elements $z \in \mathfrak{A}_{n}$ in the domain $\mathfrak{D}_{s}^{*}$ of the adjoint operator $T_{*}^{*}$ if and only if the $(2 n-r) \times 2 n$ matrix $\left\|R_{r j} S_{\tau j}\right\|$ is of rank $2 n-r$ and

$$
s(Y) \Delta\left[t^{1}(Z)\right]^{*}+s^{1}(Y) \Delta[t(Z)]^{*}=0 .
$$

As shown in the proof of Theorem 4.2 , if $\Delta$ is the constant matrix satisfying (4.4) with linearly independent solutions $y_{j}$ and $z_{j}$ of $L(y)=0$ and $L^{\star}(z)$ $=0$, respectively, then $\Delta$ is nonsingular and $Z^{*}(x) K(x) Y(x)=\Delta^{-1}$. The result of the theorem is then an immediate consequence of (6.4), and the fact that by direct computation the left-hand member of (6.5) is found to be equal to $2\left[M K^{-1}(a) R^{*}-N K^{-1}(b) S^{*}\right]$.

The operator $T_{s}$ is self-adjoint if $\mathfrak{D}_{s}^{*}=\mathfrak{D}_{s}$ and $T_{s}^{*} y=T_{s} y$. If $T_{s}$ is selfadjoint then $r=n$ and, in particular, $(L(y), z)=(y, L(z))$ for $z, y \in \mathfrak{H}_{n}^{\circ}$. In view of Corollary 2 of Theorem 3.2 it follows that if $T_{s}$ is self-adjoint then $L(y)$ is an SL-operator as defined in $\S 5$. Now if $L(y)$ is an SL-operator satisfying hypothesis $(\mathrm{H})$, and $\Delta$ is a constant matrix satisfying (5.2) with linearly independent solutions $y_{j}(x),(j=1, \cdots, n)$, of $L(y)=0$, then by Theorems 5.2 and 5.3 the matrix $\Delta$ is skew-Hermitian. The following result is then an immediate consequence of Theorem 6.1.

THEOREM 6.2. Suppose that $L(y)$ is an $S L$-operator satisfying hypothesis $(\mathrm{H})$, and let $T_{s}$ be the operator with $T_{s} y=L(y)$ on the domain $\mathfrak{D}_{s}$ of functions $y \in \mathfrak{A}_{n}$ satisfying (6.1), where $r=n$ and the $n \times 2 n$ matrix $\left\|M_{\sigma j} N_{\sigma j}\right\|,(\sigma, j=1, \cdots, n)$ is of rank $n$. If $y_{j}(x),(j=1, \cdots, n)$, are linearly independent solutions of $L(y)=0$, and $\Delta$ the corresponding constant matrix satisfying (5.2), then $T_{\mathrm{s}}$ is self-adjoint if and only if the $n \times n$ matrix $s^{1}(Y) \Delta[s(Y)]^{*}$ is Hermitian.

This result corresponds to the Main Theorem of Miller [9] established for systems in which $p_{\mu} \in \mathfrak{C}_{\mu},(\mu=0,1, \cdots, n)$, and Theorem 5 of Hamburger [5] for systems in which $p_{\mu} \in \Omega_{2},(\mu=0,1, \cdots, n)$. In the proofs of both Miller and Hamburger, however, in addition to hypothesis $(\mathrm{H})$ the assumption is made that the system $L(y)=0, s(y)=0$ is incompatible; the above proof shows that this assumption of incompatibility is unnecessary. 


\section{BiBLIOGRAPHY}

1. G. A. Bliss, A boundary value problem for a system of ordinary linear differential equations of the first order, Trans. Amer. Math. Soc. vol. 28 (1926) pp. 561-584.

2. M. Bôcher, Applications and generalizations of the concept of adjoint systems, Trans. Amer. Math. Soc. vol. 14 (1913) pp. 403-420.

3. E. A. Coddington and N. Levinson, Theory of ordinary differential equations, McGrawHill, 1955.

4. I. Halperin, Closure and adjoints of linear differential operators, Ann. of Math. vol. 38 (1937) pp. 880-919.

5. H. L. Hamburger, Remarks on self-adjoint differential operators, Proc. London Math. Soc. (3) vol. 3 (1953) pp. 446-463.

6. E. W. Hobson, On the fundamental lemma of the calculus of variations, and some related theorems, Proc. London Math. Soc. (2) vol. 11 (1912-1913) pp. 17-28.

7. A. Huke, An historical and critical study of the fundamental lemma of the calculus of variations, Contributions to the Calculus of Variations, 1930, University of Chicago Press, pp. (47)-(160).

8. E. Kamke, Differentialgleichungen Lösungsmethoden und Lösungen, Band I, Leipzig, 1943.

9. K. S. Miller, Self-adjoint differential systems, Quart. J. Math. Oxford Ser. (2) vol. 3 (1952) pp. 175-178.

NoRTHWESTERN UNIVERSITY, Evanston, Ill. 